[Agr. Biol. Chem., Vol. 29, No. 10, p. 967 969, 1965]

\title{
Isolation of Hydroxy Acids from Turkish Tobacco Leaves
}

Sir:

Our previous papers ${ }^{1,21}$ reported the isolation and identification of vanillic, $p$-hydroxycinnamic, $p$-hydroxybenzoic, $p$-hydroxy phenylacetic, caffeic, protocatechuic, azelaic, $\mathrm{D}-\beta$ phenyllactic acid and 2-isopropylmalic acids and the estimation of feluric acid in the acid fraction of Turkish tobacco leaves.

This paper deals with the isolation of $\alpha$ hydroxyisovaleric, $\alpha$-hydroxyisocaproic and $\beta$ hydroxyisocaproic acids.

The acid fraction of the ether extract obtained from Turkish tobacco leaves (Izumir, 1962 crop) was decolorized with the charcoal and chromatographed on ion exchange resin to remove phenolic acids as described in the previous paper. ${ }^{2}$ The phenolic acid-free fraction thus obtained was esterified with diazomethane and distilled. A distillate (b.p. $42 \sim 132^{\circ} \mathrm{C} / 4 \mathrm{mmHg}$ ) was saponified and then chromatographed on columns, which were prepared by mixing $40 \mathrm{~g}$ of silicic acid with $23 \mathrm{ml}$ of $0.1 \mathrm{~N}$ sulfuric acid, using chloroform and mixtures of chloroform and tert-butanol as developer. A fraction eluted with tert-butanol-chloroform $(1: 19)$ was rechromatographed. Two fractions $\mathrm{A}$ and $\mathrm{B}$ eluted respectively with tert-butanol-chloroform (1:99) and tertbutanol-chloroform $(1: 49)$ were esterified, and preliminarily separated into two parts trapped within and after ten minutes by the preparative gas chromatographic procedure under the same conditions as described in (II) of Table I. The gas chromatograms of the methyl esters trapped within ten minutes from $\mathrm{A}$ and $\mathrm{B}$ are shown in Fig. $1 \mathrm{a}$ and $\mathrm{lb}$, respectively. Each fraction corre sponding each peak in Fig. 1 was purified gas chro-

1) T. Fukuzumi, H. Takahara, H. Kaneko and I. Onishi, J. Agr. Chem. Soc. Japan, 39, 199 (1965).

2) T. Fukuzumi, H. Takahara, H. Kaneko and I. Onishi, ibid., 39, 204 (1965).
Table I. Comparison of Relative Retention Times of Peaks 2, 3, AND 5 WITH Those of THE AUThENTIC HydRoxy ACIDS

$$
\text { Sample }
$$

Peak 2

Relative retention time*

$\begin{array}{lll}\alpha \text {-Hydroxyisocaproic acid } & 0.28 & 0.23 \\ \text { Peak 3 } & 0.22 & 0.16 \\ \alpha \text {-Hydroxyisovaleric acid } & 0.22 & 0.16 \\ \text { Peak 5 } & 0.33 & 0.32 \\ \beta \text {-Hydroxyisocaproic acid } & 0.33 & 0.32\end{array}$

* Calcd. as methyl 2-isopropylmalate $=1.00$

(I) Column: $20 \%$ PEG 6000 on Shimalite W $(4 \mathrm{~mm} \times 3 \mathrm{~m})$; Column temp.: $170^{\circ} \mathrm{C}$; Carrier gas: $\mathrm{He}, 35 \mathrm{ml} / \mathrm{min}$.

(II) Column: $20 \%$ Succinate polyester on Shimalite ( $4 \mathrm{~mm} \times$ $3 \mathrm{~m})$

Column temp.: $170^{\circ} \mathrm{C}$; Carrier gas: $\mathrm{He}, 35 \mathrm{ml} / \mathrm{min}$.

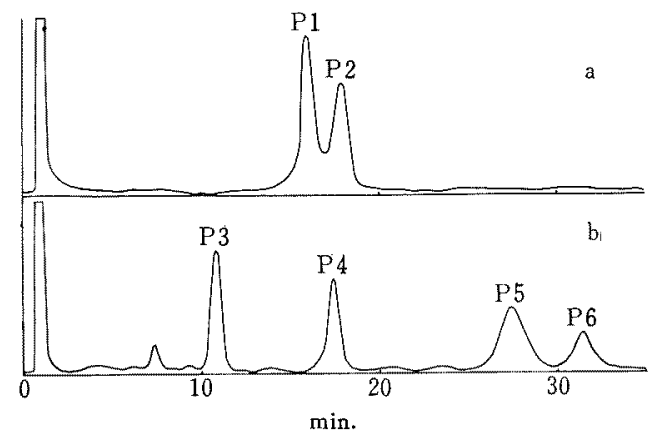

FIG. 1. Gas Chromatograms of the Methyl Esters Trapped within 10 Minutes from $A$ and $B$.

1a: Trapped from A; 1b: Trapped from B.

Conditions. Column: $20 \%$ Succinate polyester on Shimalite $W(4 \mathrm{~mm} \times 3 \mathrm{~m})$; Column temp. $130^{\circ} \mathrm{C}$; Carrier gas: He, $40 \mathrm{ml} / \mathrm{min}$.

matographically.

The infrared spectra of the samples corresponding to peaks 2,3 and 5 were identical with those of the authentic $\alpha$-hydroxyisocaproic, $\alpha$-hydroxyisovaleric and $\beta$-hydroxyisocaproic acids as shown in Fig. 2. Relative retention time (methyl 2-isopropylmalate $=1.00$ ) of each sample was also the same as that of the 


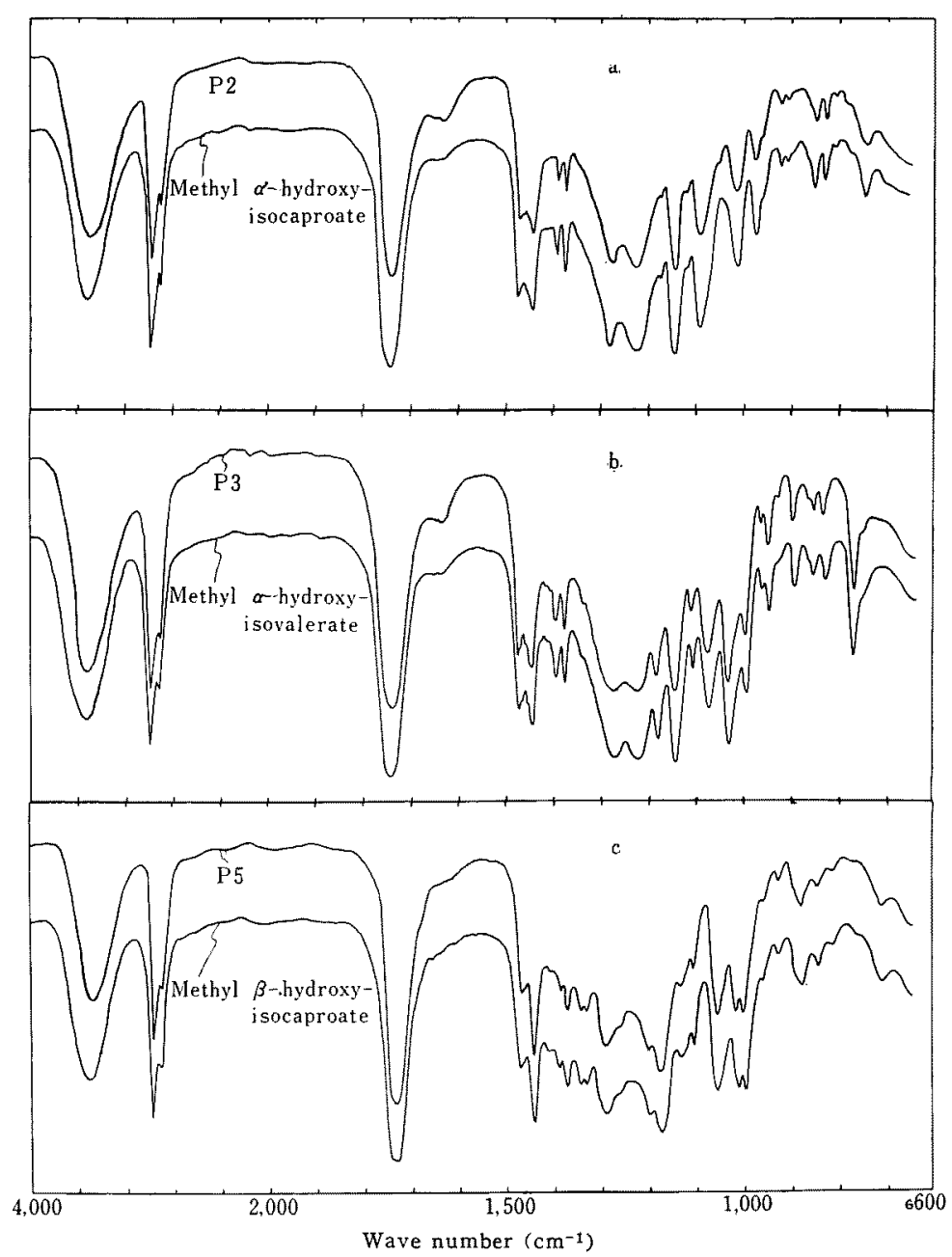

FIG. 2. Infrared Spectra of Compounds Corresponding to Peaks 2, 3 and 5 and Authentic Hydroxy Acids.

authentic one, respectively, as shown in Table I.

$\beta$-Hydroxyisocaproic acid probably has an important effect upon the aroma of Turkish Tobacco because even a small amount of the acid gives off fragrant and sweet smell. This acid might be formed by the decarboxylation of 2-isopropylmalic acid which was found previously in the acid fraction of Turkish tobacco leaves.

$\alpha$-Hydroxyisocaproic and $\alpha$-hydroxyisovaleric acids also seem to exert an influence on the aroma of Turkish tobacco smoke.

We don't know any report, have not reported, so far, concerning the presence of all these acids in tobacco leaves. Identification of peaks 1 and 4 in Fig. 1 is in progress and these studies will be published in detail 
elsewhere.

Tetsuo Fukuzumi Hiroyasu Takahara

Hajime KaneKo

Isao $\overline{\mathrm{O}}_{\mathrm{NISHI}}$
The Central Research Institute, Japan Monopoly Corporation, Tokyo

Received August 19, 1965 